\title{
A combinatorial formula for the signature of alternating diagrams
}

\author{
by
}

\section{Paweł Traczyk (Warszawa)}

Abstract. We express the signature of an alternating link in terms of some combinatorial characteristics of its diagram.

This short note is a new version of the author's unpublished manuscript $[\mathrm{T}]$. Our purpose is to give a simple combinatorial formula for the (Murasugi) signature of an alternating link in terms of combinatorial characteristics of an alternating diagram.

Let $D$ be an alternating diagram of an oriented link $L$. For simplicity we assume that $D$ is connected and reduced. We recall that a diagram is reduced if it contains no nugatory crossing (see Figure 1).

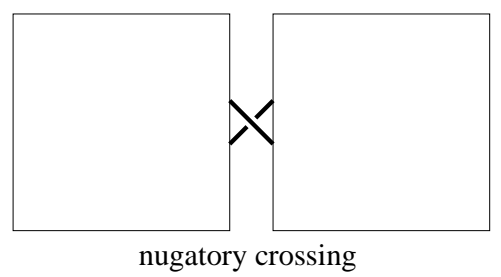

Fig. 1

A diagram that does contain a nugatory crossing may be simplified in an obvious way. The simplified diagram is still alternating. Every crossing in a diagram of an oriented link is positive (Figure 2a) or negative (Figure $2 b$ ).

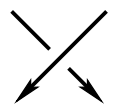

a

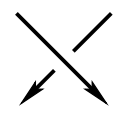

b

Fig. 2

2000 Mathematics Subject Classification: 57M25, 57M27.

Supported by KBN grant No 1 P03A 00526. 
A crossing in an oriented diagram may be smoothed as in Figure 3.

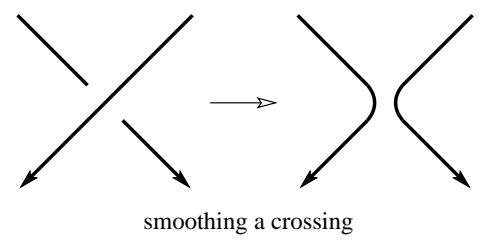

Fig. 3

If a crossing of an alternating diagram is smoothed the resulting diagram is still alternating. The formula we are about to formulate is, in essence, based on the following observation.

Theorem 1. Let $D_{0}$ be obtained from $D$ by smoothing one non-nugatory crossing $c$ of sign $\operatorname{sgn}(c)= \pm 1$. Then $\sigma\left(D_{0}\right)=\sigma(D)+\operatorname{sgn}(c)$.

Obviously, a connected diagram with all crossings nugatory represents the trivial knot, so the signature is equal to 0 . Now, one can use Theorem 1 to calculate the signature of an alternating link step by step.

We will not prove Theorem 1 directly. Instead, we will formulate a theorem providing a ready-to-use combinatorial formula for the signature of an alternating link. We will then prove the theorem using some facts that are given in the classical papers [M2] of Murasugi and [K] of Kauffman. In the Appendix we give a more direct proof of Theorem 1.

We need to prepare some terminology. The Seifert graph of a diagram $D$ is a signed (planar) graph $\Gamma$ whose vertices correspond to Seifert circles of the diagram and edges correspond to crossings of the diagram. The sign of an edge is determined by the sign of the corresponding crossing. As seen from the definition, multiple edges are allowed. Let $c_{+}$(resp. $c_{-}$) be the number of positive (resp. negative) crossings of $D$. Then $w=c_{+}-c_{-}$is the writhe or twist number of $D$. Let $T$ be a spanning tree of $\Gamma$. This means that $T$ is a tree contained in $\Gamma$ such that all vertices of $\Gamma$ are vertices of $T$. Let $d_{+}$(resp. $d_{-}$) be the number of positive (resp. negative) edges of the graph $T$.

For yet another formulation of the theorem we recall the convention for chess-board shading of the projection plane. In an alternating diagram we choose the standard shading as in Figure 4a as opposed to the shading given in Figure 4b.

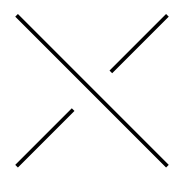

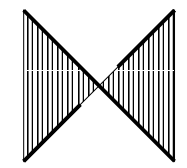

a

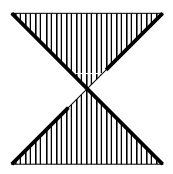

b

Fig. 4 
We denote by $B$ the number of black (shaded) areas and by $W$ the number of white areas.

We will prove the following theorem.

THEOREM 2. If $D$ is a reduced alternating diagram of an oriented link, then

$$
\begin{aligned}
& \sigma(D)=-\frac{1}{2}\left(c_{+}-c_{-}\right)+d_{+}-d_{-}=-\frac{1}{2} w+d_{+}-d_{-}, \\
& \sigma(D)=-\frac{1}{2}\left(c_{+}-c_{-}\right)+\frac{1}{2}(W-B)=-\frac{1}{2} w+\frac{1}{2}(W-B) .
\end{aligned}
$$

Proof. We will prove (2) as a simple consequence of some results from [M2] and [K]. First of all, by the Theorem of [M2] we have

$$
d_{\max } V+d_{\min } V=w-\sigma,
$$

where $V$ is the Jones polynomial of the link represented by the reduced alternating diagram $D$. It follows that

$$
\sigma=w-\left(d_{\max } V+d_{\min } V\right) .
$$

Now we need to express $d_{\max } V+d_{\min } V$ in terms of combinatorial parameters of the diagram. First we quote the following formulas given in $[\mathrm{K}]$ (where $f$ means the Kauffman version of the Jones polynomial, so that $\left.V(t)=f\left(t^{-1 / 4}\right)\right)$ :

$$
\begin{gathered}
d_{\max } f=-3 w+c+2(W-1), \\
d_{\min } f=-3 w-c-2(B-1)
\end{gathered}
$$

Adding the above two equalities we obtain

$$
d_{\max } f+d_{\min } f=-6 w+2(W-B) .
$$

Now we go back to the $V$ polynomial:

$$
d_{\max } V+d_{\min } V=\frac{3}{2} w-\frac{1}{2}(W-B) .
$$

Putting (3) and (4) together we obtain (2).

Theorem 1 follows easily: If a positive non-nugatory crossing is split, then the writhe decreases by 1 , the number of black areas decreases by one and the number of white areas does not change. It follows that the signature (as calculated from formula (2)) increases by 1. A similar argument applies to a negative crossing. This completes the proof of Theorem 1. Part (1) of Theorem 2 follows easily from Theorem 1 .

Appendix. We will now give a more direct proof of Theorem 1. The (Murasugi) signature of an oriented link $L$ is defined as the signature of the symmetric matrix $M=S+S^{T}$, where $S$ is the Seifert matrix of the link. 
Call $\operatorname{Det}_{L}=\operatorname{det}(i M)$ the determinant of $L$. It is clear that $\operatorname{Det}_{L}$ is either real or imaginary (possibly zero). Also, $\operatorname{Det}_{L}=\nabla_{L}(-2 i)=f\left(\frac{1+i}{\sqrt{2}}\right)$, where $\nabla$ is the Conway polynomial and $f$ is the Kauffman version of the Jones polynomial. It is an elementary observation that if $\operatorname{Det}_{L} \neq 0$, then the $\bmod$ 4 value of the signature of $L$ is determined by $\operatorname{Det}_{L}$. Namely, $\sigma$ is congruent modulo 4 to $0,1,2$ or 3 when $\operatorname{Det}_{L}$ is positive real, positive imaginary, negative real or negative imaginary respectively. This is equivalent to saying that

$$
i^{\sigma}=\frac{\text { Det }}{\mid \text { Det } \mid} .
$$

It is known that if $L_{0}$ is obtained from $L$ by smoothing a single crossing, then $\sigma(L)$ and $\sigma\left(L_{0}\right)$ differ by at most one. In Theorem 1 we claim that if $L_{0}$ and $L$ are represented by alternating diagrams $D_{0}$ and $D$, then specifically

$$
\sigma\left(D_{0}\right)-\sigma(D)=\operatorname{sgn}(c) .
$$

Assume that the sign of $c$ is positive. We need to show that $\sigma\left(D_{0}\right)-\sigma(D)=1$. It will be sufficient to show that $\operatorname{Det}_{L_{0}}$ and $\operatorname{Det}_{L}$ are both non-zero and that the oriented angle from the vector (in the complex plane) $\operatorname{Det}_{D_{0}}$ to $\operatorname{Det}_{D}$ is $-\pi / 2$. This is equivalent to saying that $\operatorname{Det}_{D_{0}}=r \cdot i \cdot \operatorname{Det}_{D}$ or that

$$
f_{D_{0}}(\theta)=r \cdot i \cdot f_{D}(\theta),
$$

where $\theta=(1+i) / \sqrt{2}$ and $r$ is positive real. Let $w$ be the writhe of $D$ so that $w-1$ is the writhe of $D_{0}$. Now, the idea is to work with unoriented diagrams. For a given unoriented diagram $D$ with a chosen crossing $c$ we will denote by $D_{0+}$ and $D_{0-}$ the two diagrams shown in Figure 5 .

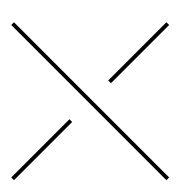

$\mathrm{D}$

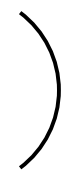

$\mathrm{D}_{0-}$

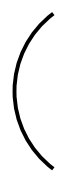

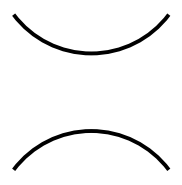

$\mathrm{D}_{0+}$

Fig. 5

In this notation the sign refers to local orientation of the crossing that would give a particular type of smoothing (e.g. $D_{0+}$ is obtained in the following way: first we choose a local orientation of the crossing that makes this crossing positive, then we smooth this crossing according to the orientation). Let us stress that only local orientation is needed and it may well be that there is no global orientation giving the prescribed sign to a given crossing. To reformulate the problem in terms of unoriented diagrams let us 
rewrite (5) in terms of the Kauffman bracket (see [K, p. 399]):

$$
(-\theta)^{-3 w} \cdot\left\langle D_{0+}\right\rangle(\theta)=r \cdot i \cdot(-\theta)^{-3 w+3} \cdot\langle D\rangle(\theta) .
$$

This is equivalent to

$$
\left\langle D_{0+}\right\rangle(\theta)=r \cdot i \cdot(-\theta)^{3} \cdot\langle D\rangle(\theta)
$$

and finally to

$$
\left\langle D_{0+}\right\rangle(\theta)=r \cdot \theta \cdot\langle D\rangle(\theta)
$$

(because $\theta^{2}=i$ ). We will show that for any alternating connected dia$\operatorname{gram} D$,

$$
\langle D\rangle(\theta)=s \cdot \theta^{B-W},
$$

where $s$ is a positive real number. Once this is proved, (6) follows immediately.

Now we prove (7). If all crossings of $D$ are nugatory, then $D$ represents the trivial knot. It can be given an orientation. The orientation then defines the signs of crossings. The signs are uniquely determined, no matter how the orientation is chosen. It is easy to see that in such a situation $c_{+}=W$ and $c_{-}=B$. Since $1=f_{D}=(-A)^{-3 w}\langle D\rangle=(-A)^{-3 W+3 B}\langle D\rangle$ it follows that $\langle D\rangle=(-A)^{3 W-3 B}$, in particular $\langle D\rangle(\theta)=(-\theta)^{3 W-3 B}=\theta^{B-W}$, as required. The inductive step is trivial: If $D$ has a non-nugatory $\operatorname{crossing} c$, then

$$
\langle D\rangle(\theta)=\theta^{-1}\left\langle D_{0-}\right\rangle(\theta)+\theta\left\langle D_{0+}\right\rangle(\theta) .
$$

By the inductive assumption

$$
\langle D\rangle(\theta)=\theta^{-1} t \cdot \theta^{B-W+1}+\theta \cdot u \cdot \theta^{B-W-1}=(s \theta)^{B-W},
$$

which completes the proof of (6) and of Theorem 1.

Final remark. We have given a simple proof of Theorem 1. In a way, this theorem may be seen as a principle governing the behaviour of the signature of alternating links. Some classical results follow by simple combinatorics. For example it is easily proved that for alternating diagrams the signature is additive with respect to the $\star$ product $([\mathrm{M} 1$, Theorem 5.4]).

\section{References}

[K] L. H. Kauffman, State models and the Jones polynomial, Topology 26 (1987), 395-407.

[M1] K. Murasugi, On a certain numerical invariant of link types, Trans. Amer. Math. Soc. 117 (1965), 387-422.

[M2] - Jones polynomials and classical conjectures in knot theory II, Math. Proc. Cambridge Philos. Soc. 102 (1987), 187-194. 
[T] P. Traczyk, A combinatorial formula for the signature of alternating diagrams, unpublished, 1987.

Department of Mathematics

Warsaw University

Banacha 2

02-097 Warszawa, Poland

E-mail: traczyk@mimuw.edu.pl

Received 29 June 2004;

in revised form 25 July 2004 